\title{
Luteoloside Prevents Lipopolysaccharide-Induced Osteolysis and Suppresses RANKL-Induced Osteoclastogenesis Through Attenuating RANKL Signaling Cascades
}

Fangming Song 1,2,\#, Chengming, Wei ${ }^{1, \#}$, Lin Zhou ${ }^{2}$, Qin $\mathrm{An}^{4}$, Mingli Yang ${ }^{2}$, Jennifer Tickner ${ }^{2}$, Yuanjiao Huang ${ }^{3}$, Jinmin Zhao ${ }^{1,5, *}$ and Jiake $\mathrm{Xu}^{1,2,{ }^{*}}$

${ }^{1}$ Research Centre for Regenerative Medicine, Guangxi Key Laboratory of Regenerative Medicine, Guangxi Medical University, Nanning, Guangxi, China

${ }^{2}$ School of Pathology and Laboratory Medicine, The University of Western Australia, Perth, Western Australia, Australia

${ }^{3}$ Medical Scientific Research Center, Guangxi Medical University, Nanning, Guangxi, China

${ }^{4}$ Department of Orthopaedics, Shanghai Key Laboratory of Orthopaedic Implant, Shanghai Ninth People's Hospital, Shanghai Jiaotong University School of Medicine, Shanghai, China

${ }^{5}$ Department of Orthopaedic Surgery, The First Affiliated Hospital of Guangxi Medical University, Nanning, Guangxi, China

\#These authors contributed equally to this work.

* Correspondence to: Jinmin Zhao, Research Centre for Regenerative Medicine, Guangxi Key Laboratory of Regenerative Medicine, Department of Orthopaedic Surgery, The First Affiliated Hospital of Guangxi Medical University, Guangxi Medical University, Guangxi, China

Email: ZJinMin@hotmail.com

* Correspondence to: Jiake Xu, Research Centre for Regenerative Medicine, Guangxi Key Laboratory of Regenerative Medicine, Guangxi Medical University, Nanning, Guangxi, China; School of Pathology and Laboratory Medicine, The University of Western Australia, Perth, Western Australia, Australia

E-mail: jiake.xu@uwa.edu.au. 


\begin{abstract}
Bone destruction or osteolysis marked by excessive osteoclastic bone resorption is a very common medical condition. Identification of agents that can effectively suppress excessive osteoclast formation and function is crucial for prevention and treatment of osteolytic conditions such as periprosthetic joint infection and periprosthetic loosening. Luteoloside, a flavonoid, is a natural bioactive compound with anti-inflammation and anti-tumor properties. However, the effect of Luteoloside on inflammation-induced osteolysis is unknown. Here, we found that Luteoloside exhibited a strong inhibitory effect on lipopolysaccharide (LPS)-induced osteolysis in vivo. In addition, Luteoloside suppressed RANKL-induced osteoclast differentiation and abrogated bone resorption in a dose-dependent manner. Further, we found that the anti-osteoclastic and anti-resorptive actions of Luteoloside are mediated via blocking NFATc1 activity and the attenuation of RANKL-mediated $\mathrm{Ca}^{2+}$ signaling as well as NF- $\mathrm{BB}$ and MAPK pathways. Taken together, this study shows that Luteoloside may be a potential therapeutic agent for osteolytic bone diseases associated with abnormal osteoclast formation and function in inflammatory conditions.
\end{abstract}

KEY WOREDS: LUTEOLOSIDE; OSTEOCLAST; OSTEOLYSIS 


\section{Introduction}

Bone destruction or osteolysis characterized by excessive osteoclastic bone resorption is commonly present in patients of periprosthetic joint infection and periprosthetic loosening, which could affect the treatment outcome of orthopedic procedures. For instance, total joint arthroplasty (TJA) is considered an effective surgical treatment available to help patients with pain and mobility suffering from severe joint diseases, such as osteoarthritis and rheumatoid arthritis ${ }^{[1]}$. However, the overall outcome of TJA is greatly influenced by periprosthetic joint infection and periprosthetic osteolysis, which could result in aseptic loosening and revision surgery ${ }^{[2,3]}$. In addition, bacterial endotoxin contamination or adherent bacterial endotoxin on implant-derived wear particles is a major cause of inflammatory osteolysis due to abnormally enhanced osteoclastic bone resorption ${ }^{[4-6]}$. Furthermore, Lipopolysaccharide (LPS), a classical bacterial endotoxin, is believed to be the common trigger of bone loss accompanying enhanced osteoclastic activity ${ }^{[7,8]}$.

Of note, pathological bone conditions initiated by LPS exhibit recruitment of a number of cell types, such as macrophages, fibroblasts and osteoclasts, followed by eliciting inflammatory cytokine secretion, including TNF-a, IL-1, IL-6, and M-CSF ${ }^{[9}$, 10]. Furthermore, activation of nuclear factor- $\kappa \mathrm{B}$ ligand (RANKL) contributes to osteolysis by favoring exacerbated osteoclast activity and accelerating bone resorption in response to inflammatory cytokines ${ }^{[5]}$. Given the crucial role of excessive osteoclastic activity in bone destruction, development of osteoclastic inhibitors can offer valuable information for the prevention and treatment of pathological osteolysis such as periprosthetic joint infection and periprosthetic loosening.

Luteoloside, a flavonoid that can be isolated from the plant Lonicera japonica, exerts biomedical and pharmacological activities, including anti-tumor ${ }^{[11]}$, anti-bacterial $^{[12]}$, anti-inflammation ${ }^{[13]}$, and antiviral properties ${ }^{[14]}$. Interestingly, previous studies have reported that Luteoloside exhibited an inhibitory effect on 
LPS-induced inflammatory responses, suggesting that it may be potentially useful for the treatment of bone diseases associated with LPS activation ${ }^{[13]}$.

In this study, we investigated the in vivo effect of Luteoloside on LPS-induced bone erosion, and elucidated its cellular and molecular mechanism of action in vitro. Our results demonstrate that Luteoloside can prevent LPS-mediated bone loss in vivo. Furthermore, our finding also showed that Luteoloside could inhibit osteoclastogenesis and bone resorption via the suppression of NFATc1 activity related to $\mathrm{Ca}^{2+}$ signaling.

\section{Materials and Methods}

Materials and reagents

Alpha modified Minimal Essential Medium ( $\alpha$-MEM) and fetal bovine serum (FBS) were obtained from Thermo Fisher Scientific (Scoresby, Vic, Australia). Luteoloside of purity $>95 \%$ was purchased from Chengdu Must Bio-Technology Co., Ltd (Chengdu, Sichuan Province, China) and dissolved in Dimethyl sulfoxide (DMSO) at a concentration of $100 \mu \mathrm{M}$. Anti-ERK, anti-phospho-ERK, anti-JNK, anti-phospho-JNK, anti-p38, anti-phospho-p38, anti-IкB $\alpha$, anti-p65, anti-NFATc1, anti-c-Fos and anti-ß-Actin antibodies were obtained from Santa Cruz Biotechnology (Santa Cruz, CA, USA). Vacuolar-type H+-ATPase V0 subunit d2 (V-ATPase d2) was generated as previously described ${ }^{[15]}$. The MTS assay kit and luciferase assay system were obtained from Promega (Madison, WI, USA). Leucocyte acid phosphatase staining kits were obtained from Sigma-Aldrich (St Louis, MO, USA). Recombinant macrophage colony stimulating factor (M-CSF) was obtained from R\&D Systems (Minneapolis, MN, USA). Recombinant GST-rRANKL protein was expressed and purified as previously described ${ }^{[16]}$. Streptavidin-horseradish peroxidase and Dako Liquid DAB were purchased from DAKO (Carpinteria, CA, USA). ProLong Diamond anti-fade mounting medium were obtained from Invitrogen (Carlsbad, CA, USA). Rhodamine-conjugated phalloidin was purchased from Molecular Probes, Inc. (Eugene, OR, USA). DAPI was purchased from Santa Cruz Biotechnology (Santa Cruz, CA, USA). 


\section{Ethical Use of Animals}

All procedures utilizing mice were performed in accordance with the National Health and Medical Research Council of Australia 'Guidelines to promote the wellbeing of animals used for scientific purposes: The assessment and alleviation of pain and distress in research animals (2008)', in conjunction with the 'Australian code for the care and use of animals for scientific purposes 8th edition (2013)'; and also in accordance with the guidelines for 'Ethical Conduct in the Care and Use of Nonhuman Animals in Research' by the American Psychological Association. All protocols were approved by the UWA animal ethics committee (approval RA/3/100/1244) or the Animal Care and Welfare Committee of Guangxi Medical University (SYXK2009-0004).

In vitro osteoclastogenesis assay

Bone marrow macrophage cells (BMMs) were isolated from six-week-old C57BL/6J mice by flushing the marrow from the femur and tibia. Cells were then cultured in a-MEM supplemented with 10\% FBS, 2 mM L-glutamine, $100 \mathrm{U} / \mathrm{ml}$ penicillin, and $100 \mu \mathrm{g} / \mathrm{ml}$ streptomycin (complete medium), in the presence of M-CSF (50 ng/mL). To generate osteoclasts, BMMs were plated in 96-well plates at a density of $6 \times 10^{3}$ cells/well in the presence of M-CSF (50 ng/mL) overnight. The following day, cells were then stimulated with complete medium containing M-CSF and GST-rRANKL (50 ng/mL) in the presence or absence of Luteoloside $(0.25,0.5,1,2.5,5,10 \mu \mathrm{M})$ every 2 days until osteoclasts formed. After 5 days, cells were fixed with $4 \%$ paraformaldehyde and then stained for tartrate resistant acid phosphatase (TRAcP) enzymatic activity using a leucocyte acid phosphatase staining kit, following the manufacturer's procedures. TRAcP-positive multinucleated cells (>3 nuclei) were scored as osteoclast-like (OCL) cells. In addition, the effects of Luteoloside (10 $\mu \mathrm{M})$ at different stages of osteoclast differentiation was also investigated. Cells were cultured with M-CSF and RANKL and then supplemented with Luteoloside at the early stage (added on day 1 and removed on day 3), mid stage (added on day 3 and removed on day 5), or late stage (added on day 5 and removed on day 6) of osteoclast 
culture or whole treatment period (added on day 1, day 3, day 5 and removed on day 6). Finally, plates were fixed and stained for TRAcP as described above.

\section{Cytotoxicity assay}

BMMs were seeded in 96-well plates at $6 \times 10^{3}$ cells/well and incubated overnight to adhere. Next day, cells were treated with Luteoloside (0, 0.25, 0.5, 1, 2.5, 5, $10 \mu \mathrm{M})$ for $48 \mathrm{~h}$. MTS solution (20 $\mu \mathrm{L} /$ well) was then added to each well, and cells were incubated for an additional $2 \mathrm{~h}$. The absorbance at $490 \mathrm{~nm}$ was read with a microplate reader (Thermo, USA).

Immunofluorescent staining and confocal microscopy

BMMs were seeded at a density of $6 \times 10^{3}$ cell/well in the presence of M-CSF (50 ng/mL) overnight. Cells were stimulated with M-CSF and GST-rRANKL (50 ng/mL) in the presence of Luteoloside until mature osteoclasts formed. Cells were fixed with 4\% paraformaldehyde, permeabilized with $0.1 \%$ Triton X-100 PBS, and then blocked with 3\% BSA in PBS. Cells were then incubated with Rhodamine-conjugated phalloidin for 45 minutes in the dark to stain F-actin. Cells were then washed with PBS, stained with DAPI, and mounted for confocal microscopy.

Hydroxyapatite resorption assay

BMMs $\left(1 \times 10^{5}\right.$ cells/well $)$ were cultured onto 6-well collagen-coated plates and stimulated with GST-rRANKL and M-CSF (50 ng/mL) until mature osteoclasts formed. Cells were gently harvested using cell dissociation solution (Sigma Aldrich, St Louis, MO, USA) and consistent numbers of mature osteoclasts were seeded into hydroxyapatite-coated 96 well plates (Corning, USA). Mature osteoclasts were incubated in medium containing GST-rRANKL and M-CSF with or without Luteoloside. After $48 \mathrm{~h}$, half of the wells were stained with TRAcP for osteoclast counting and the remaining wells were bleached to remove cells, followed by image acquisition for the measurement of resorbed areas using a Nikon Ti-U inverted microscope (Nikon, Melville, NY, USA). The percentage of surface resorbed was analyzed using Image J software (NIH, Bethesda, USA).

Luciferase reporter assay 
RAW264.7 cells stably transfected with either an NF-kB-responsive luciferase construct $^{[17]}$ or an NFATc1-responsive luciferase reporter construct ${ }^{[18]}$ were seeded in 48-well plates at a density of $1.5 \times 10^{5}$ cells/well or $5 \times 10^{4}$ cells/well overnight respectively. The next day, cells were pretreated with Luteoloside for $1 \mathrm{~h}$. Following pre-treatment cells were subsequently stimulated with GST-rRANKL (50 ng/mL) for $6 \mathrm{~h}$ (NF-кB luciferase reporter gene assay) or $24 \mathrm{~h}$ (NFATc1 luciferase reporter gene assay) in the presence of Luteoloside and luciferase activity was measured using the luciferase reporter assay system according to the manufacturer's protocol (Promega, Sydney, Australia).

Quantitative real-time RT-PCR

Total RNA was isolated from cells using Trizol reagent according to the manufacturer's protocol (ThermoFisher Scientific, Scoresby, Australia). The cDNA was synthesized using Moloney murine leukemia virus reverse transcriptase with $1 \mu \mathrm{g}$ of RNA template and oligo-dT primers. The resultant cDNA was then utilized for SYBR Green-based real-time PCR. Expression levels were normalized to GAPDH expression and fold change determined using Livak's equation. The following specific primers (based on the mouse sequences) were used:

Cathepsin K (CTSK) (Forward: 5'-GGGAGAAAAACCTGAAGC-3'; Reverse:

5'-ATTCTGGGGACTCAGAGC-3'), Calcitonin receptor (Forward:

5'-TGGTTGAGGTTGTGCCCA-3'; Reverse: 5'-CTCGTGGGTTTGCCTCATC-3';), V-ATPase-d2 (Forward: 5'-GTGAGACCTTGGAAGACCTGAA-3'; Reverse:

5'-GAGAAATGTGCTCAGGGGCT-3'), MMP-9 (Forward:

5'-CGTGTCTGGAGATTCGACTTGA-3'; Reverse:

5'-TTGGAAACTCACACGCCAGA-3'), NFATc1 (Forward:

5'-CAACGCCCTGACCACCGATAG-3'; Reverse:

5'-GGCTGCCTTCCGTCTCATAGT-3'), TRAcP (Acp5)(Forward:

5'-TGTGGCCATCTTTATGCT-3'; Reverse: 5'-GTCATTTCTTTGGGGCTT-3'),

,GAPDH (Forward: 5'-ACCACAGTCCATGCCATCAC-3';Reverse:

5'-TCCACCACCCTGTTGCTGTA-3').

Western blotting 
BMM cells were cultured in 6-well plates and stimulated with GST-rRANKL and M-CSF for the stated times. Cells were lysed in radioimmunoprecipitation (RIPA) lysis buffer, and proteins were separates using SDS-polyacrylamide gel electrophoresis and transferred to polyvinylidene fluoride (PVDF) membranes (GE Healthcare, Australia). The membranes were blocked with 5\% skim milk for $1 \mathrm{~h}$ before being probed with specific primary antibodies with gentle shaking overnight. Membranes were washed and subsequently incubated for $1 \mathrm{~h}$ with horseradish peroxidase (HRP)-conjugated secondary antibodies, followed by detection using enhanced chemiluminesence (ECL) reagent (Amersham Pharmacia Biotech, Australia). Protein bands were visualized on an Image-quant LAS 4000 (GE Healthcare, Australia) and analyzed by Image J software.

p65 immunohistochemistry

BMMs $\left(6 \times 10^{3}\right.$ cell/well $)$ were seeded in 96-well plates overnight. The cells were pre-incubated with Luteoloside for $1 \mathrm{~h}$, followed by stimulated with GST-rRANKL (100 ng/mL) for $30 \mathrm{~min}$. Cells were fixed with 4\% paraformaldehyde, permeabilized with $0.1 \%$ Triton $\mathrm{X}-100 \mathrm{PBS}$, and then incubated in the presence or absence of anti-p65. Cells were washed with 0.1\% BSA-PBS and PBS before adding Streptavidin-horseradish peroxidase-conjugated secondary antibody. Finally, Dako Liquid DAB was add for 30 min or until brown coloration appeared.

Measurement of intracellular $\mathrm{Ca}^{2+}$ oscillation

BMMs were seeded into a 48-well plate at a concentration of $1 \times 10^{4}$ cells/well for 24 h. The following day, the medium was changed with complete medium containing 10 $\mu \mathrm{M}$ Luteoloside supplemented with GST-rRANKL and M-CSF for $24 \mathrm{~h}$. Cells were then rinsed with Assay buffer (HANKS balanced salt solution supplemented with 1 $\mathrm{mM}$ probenecid and 1\% FBS), followed by loading with $4 \mu \mathrm{M}$ Fluo4 staining solution (Fluo4-AM dissolved in 20\% pluronic-F127 (w/v) in DMSO diluted in Assay buffer) for $45 \mathrm{~min}$. The cells were washed once in Assay buffer and incubated at room temperature for 20 minutes followed by a further 2 washes in Assay buffer. The intensity of fluorescence was viewed on an inverted fluorescent microscope (Nikon Ti-U) excited at $488 \mathrm{~nm}$ and analyzed using Nikon Basic Research Software. Images were captured at 2 second intervals for 3 minutes. Oscillating cells showed more than 
1 calcium peak within the observed time frame, with the oscillation intensity calculated as the difference between the maximum and minimum of fluorescence intensities within the oscillating cell area.

In vivo calvarial model of LPS-induced osteolysis

The animal experiments were approved by Institutional Animal Ethics Committee of Guangxi Medical University (SCXK-(JUN) 2012-0004, Beijing, China). Twenty 8-week-old C57/BL6 mice were divided into four groups containing five mice: Sham group (PBS; control), Vehicle group (LPS; $5 \mathrm{mg} / \mathrm{kg}$ body weight), LPS with low dose Luteoloside (1 mg/kg) and LPS with high dose Luteoloside (2 mg/kg). Mice were injected with Luteoloside or PBS 1 day before injection of LPS. Seven days after LPS injection, all mice were sacrificed and the calvarias were dissected and fixed in $4 \%$ paraformaldehyde for micro-CT and histological analysis.

\section{Micro-CT scanning}

The three-dimensional images of whole calvaria were reconstructed using a high resolution micro-CT ( $\mu \mathrm{CT}$ ) scanner (Skyscan 1176; Skyscan; Aartselaar, Belgium). Images were acquired using $50 \mathrm{kV}$ X-ray tube voltage, $800 \mu \mathrm{A}$ current, an isotropic pixel size of $14.4 \mu \mathrm{M}(1024 \times 1024$ pixel image matrix $)$ and with a $0.75-m m-t h i c k$ aluminum filter for beam hardening. A square region of interest around the midline suture was chosen for further qualitative and quantitative analysis after reconstruction.

Histological and histomorphometric analysis

The specimens were decalcified with 10\% EDTA solution for 2 weeks followed by embedding into paraffin. Histological sections were prepared and staining for TRAcP activity and H\&E staining. The sections were then photographed at $40 \times$ and $100 \times$ magnifications (Nikon Eclipse TE2000-S microscope). Bone histomorphometric analyses were performed using BioQuant Osteo Histomorphometry software (Bioquant Image Analysis Corporation, Nashville, TN, USA).

Statistical analysis 
All experimental data are presented as the mean \pm SD obtained from three or more experiments and statistical significance determined by Student's t-test and One-way ANOVA. A $p$ value of less than 0.05 was considered to be significant.

\section{Results}

Luteoloside inhibits RANKL-induced osteoclastogenesis in vitro

To identify the influence of Luteoloside on RANKL-induced osteoclastogenesis, freshly isolated BMMs were incubated with RANKL and M-CSF in the presence or absence of various concentrations of Luteoloside. Increasing concentrations of Luteoloside exerted a dose-dependent inhibition on the formation of TRAcP-positive multinucleated osteoclasts (Fig. $1 A, B, C$ ). Luteoloside significantly reduced the cell size and number of RANKL-induced osteoclasts at a dose of $5 \mu \mathrm{M}$ and $10 \mu \mathrm{M}$ (Fig. 1 $D, E$ ). To examine the time course effect of Luteoloside on osteoclast differentiation, cells were exposed to Luteoloside at several different time phases. We found that osteoclast formation was reduced by Luteoloside treatment, most effectively during the early stages of osteoclast differentiation (Fig. $1 F, G$ ). To evaluate the morphological changes in mature osteoclasts affected by Luteoloside treatment, cells were stained with rhodamine-phalloidin to visualize F-actin structures in mature cells. Mature osteoclasts are shown by the appearance of well-defined podosome belts with multiple intact nuclei after RANKL stimulation. However, smaller osteoclasts with fewer nuclei were observed after Luteoloside treatment (Fig. $1 \mathrm{H}$ ). To examine whether cell cytotoxicity might be responsible for the effect of Luteoloside on suppression of osteoclast formation, MTS assay was performed to assess cell viability. We found that Luteoloside had no cell cytotoxicity on BMMs over the range of concentrations used in our study (Fig. 1 I). Taken together, these findings indicate that Luteoloside abrogates RANKL-induced osteoclastogenesis through affecting the early stages of osteoclast differentiation, but does not cause cell cytotoxicity in osteoclast precursors.

Luteoloside suppresses osteoclast hydroxyapatite resorption and osteoclastic gene expression

Next, we analyzed the effect of Luteoloside on osteoclast function using hydroxyapatite-coated plates. The area of hydroxyapatite resorption was markedly 
reduced in Luteoloside treatment groups $(5 \mu \mathrm{M}$ and $10 \mu \mathrm{M})$ compared to the RANKL only treatment group (Fig. $2 A, B, C$ ). In addition, Luteoloside down-regulated expression of resorption-related genes including CTSK, Calcitonin receptor, MMP9, TRAcP, NFATc1 and V-ATPase d2 (Fig. 2 D). Collectively, these data show that Luteoloside dose-dependently attenuates hydroxyapatite resorption in vitro.

Luteoloside abrogates NFATc1 activity and RANKL-induced calcium oscillations

To explore the molecular mechanism by which Luteoloside regulates osteoclast differentiation, we investigated the effect of Luteoloside on RANKL-mediated pathways. First, we found that Luteoloside significantly suppressed activity of NFATc1 in response to RANKL stimulation as assessed by NFAT luciferase reporter assay (Fig. 3 A). In addition, Luteoloside reduced the protein level of NFATc1, and NFATc1 downstream regulated protein V-ATPase d2. Further, the expression of c-Fos, crucial for RANKL-induced induction of NFATc1, was also attenuated by Luteoloside treatment (Fig. $3 \mathrm{~B}-E$ ). $\mathrm{Ca}^{2+}$ oscillations are stimulated by RANKL, resulted in activation of NFATc1, which is crucial for osteoclast formation. To further evaluate the effect of Luteoloside on $\mathrm{Ca}^{2+}$ oscillations, $\mathrm{Ca}^{2+}$ flux was measured following RANKL stimulation. $\mathrm{Ca}^{2+}$ flux was observed in the presence of RANKL, and the amplitude of RANKL-induced $\mathrm{Ca}^{2+}$ flux was suppressed in the presence of Luteoloside. Luteoloside reduced the intensity of $\mathrm{Ca}^{2+}$ oscillations at a concentration of $10 \mu \mathrm{M}$ (Fig. 3 F-I). Taken together, these data demonstrate that Luteoloside inhibits RANKL-induced NFATc1 activity and $\mathrm{Ca}^{2+}$ oscillations.

Luteoloside blocks NF- $\kappa$ B and MAPK signaling pathways

The induction of NFATc1 is also dependent on RANKL induced NF- $\kappa$ B signaling, and modulated by MAPK (JNK, ERK, p38) pathways. To study the role of Luteoloside on

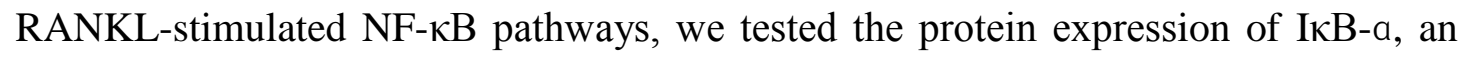

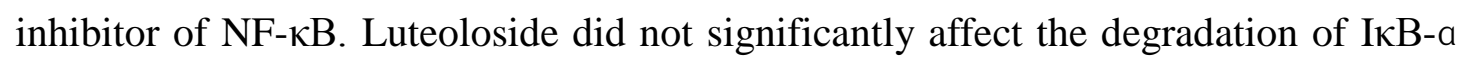
in response to RANKL stimulation (Fig. $4 A-B$ ). However, activation of NF- $\kappa B$, as measured by luciferase assay, was significantly blocked by Luteoloside (10 $\mu \mathrm{M})$ (Fig. $4 \mathrm{C}$ ). These results suggest that Luteoloside may affect p65 nuclear translocation 
downstream of IкB-a. Using immunohistochemistry, Luteoloside was indeed found to inhibit RANKL-induced p65 nuclear translocation, consistent with its ability to block NF- $\kappa B$ activation (Fig. $4 D-E$ ). To investigate the role of Luteoloside on RANKL-induced MAPK signaling pathways during osteoclast differentiation, we found that Luteoloside significantly suppressed phosphorylation of ERK and p38 relative to total ERK and total p38 respectively, but failed to block phosphorylation of JNK relative to total JNK (Fig. $4 F-I$ ). Collectively, these data show that Luteoloside inhibits NF- $\mathrm{KB}$ activity and MAPK phosphorylation in response to RANKL activation.

Luteoloside prevents LPS-induced bone loss in vivo

To further explore the effects of Luteoloside on pathological osteolysis in vivo, a mouse calvarial model of LPS-induced osteolysis was performed. Micro-CT scanning and 3D reconstruction revealed that LPS injection (Vehicle group) resulted in extensive surface erosions on the calvaria relative to Sham group (PBS injection). However, treatment with Luteoloside (low-dose group and high-dose group) strongly suppressed LPS-induced inflammatory osteolysis (Fig. 5 A). Quantitative analyses of bone parameters further demonstrated that Luteoloside treatment significantly increased the bone volume/tissue volume (BV/TV) (Fig. 5 B) and decreased the number of pores and percentage of porosity in a dose-manner dependent (Fig. 5 C-D). Histological assessment further confirmed the attenuation effect of Luteoloside on LPS-induced bone erosion. Consistent with the pronounced reduction in inflammatory osteolysis, the number of TRAcP-positive cell (arrows) observed in LPS-induced group were markedly reduced by Luteoloside treatment (arrows) (Fig. 6 A). In addition, histomorphometric analyses demonstrated that Luteoloside treatment dose-dependently increased BV/TV (Fig. 6 B) and decreased TRAcP (+) cell number and TRAcP (+) cell No/BS as well (Fig. 6 C-D). Collectively, our findings demonstrate that Luteoloside may serve as a potential and effective anti-resorptive agent in the treatment of LPS-induced osteolysis.

\section{Discussion}

In this study we observed that Luteoloside suppressed RANKL-induced osteoclast formation, bone resorption, and LPS-induced bone loss in vivo, indicative of its 
potential for the treatment of osteolysis in situations such as periprosthetic joint infection and periprosthetic osteolysis. Given the previously demonstrated anti-tumor ${ }^{[11]}$ and anti-inflammatory ${ }^{[13]}$ activities of Luteoloside, it is possible that Luteoloside might also be a drug candidate to treat osteolytic conditions concomitant with tumors and inflammation. Further uncovering the molecular and cellular mechanism of Luteoloside action will be invaluable for exploring its therapeutic potential for osteolysis.

When RANKL interacts with RANK it initiates a cascade of intracellular signaling events including NF- $\kappa$ B, MAPK, AP-1, NFATc1 and $\mathrm{Ca}^{2+}$ which are crucial for osteoclastogenesis. Among these signaling pathways, the RANKL/RANK/TRAF6 axis with costimulatory immunoreceptors results in the robust induction of transcription factors c-Fos and NFATc1, which play an indispensable role in osteoclast differentiation. c-Fos, a component of activator protein-1 (AP-1) transcription factor complex, is involved in the regulation of osteoclast formation ${ }^{[19 \text {, }}$ 20]. Targeted c-Fos mutant mice exhibit osteopetrosis, characterized by lack of osteoclasts $^{[21]}$. In this study, Luteoloside was shown to markedly down regulate the protein level of c-Fos. Furthermore, NFATc1 represents a master transcriptional factor in the differentiation of osteoclasts, functioning downstream of RANKL-mediated NF- $\kappa \mathrm{B}, \mathrm{AP}-1$ and MAPK signaling pathways ${ }^{[22]}$. Additionally, c-Fos and calcium signaling can trigger the auto-amplification of NFATc1 which is required for NFATc1-dependent transcriptional processes and RANKL-induced osteoclastogenesis ${ }^{[22,23]}$. Once NFATc1 activation is induced downstream osteoclast-specific genes are up-regulated, such as Ctsk and V-ATPase subunit d2 [24, ${ }^{25]}$. Our studies found that Luteoloside significantly attenuated mRNA and protein levels of NFATc1, followed by reduced expression of osteoclast marker genes in a dose-dependent manner. What's more, induction of NFATc1 is also dependent on $\mathrm{Ca}^{2+}$-dependent calcineurin activation ${ }^{[26]}$. In our study, we observed that Luteoloside suppressed $\mathrm{Ca}^{2+}$ oscillation induced by RANKL, in line with its inhibitory effect on NFATc1 activation. RANKL/RANK also activates the NF- $\kappa B$ pathway. In the present studies, Luteoloside was found to significantly inhibit NF- $\mathrm{B}$ activity but failed to 


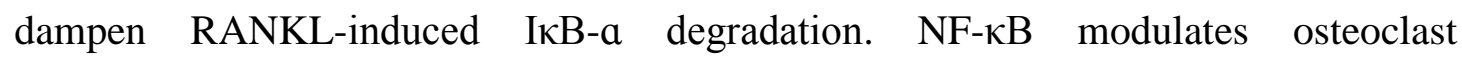
differentiation as an important transcriptional factor. The degradation of IкB-a allows

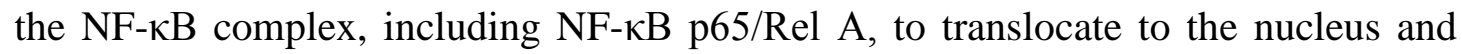
initiate the transcription of targeted genes ${ }^{[27]}$. Deletion of p65/Rel A results in severe osteopetrosis owing to deficiency in osteoclast formation ${ }^{[28,29]}$. Interestingly, we found Luteoloside was capable of preventing the nuclear translocation of p65 in response to RANKL stimulation, indicative of an effect at least in part independent on IкB-a degradation. The mitogen-activated protein kinase (MAPK) pathways (extracellular signal-regulated kinase (ERK), c-Jun N-terminal kinase (JNK), and p38 MAPK) are involved in modulation of osteoclast differentiation as well ${ }^{[30]}$. Among them, the role of ERK is linked to the survival, apoptosis and differentiation of osteoclasts ${ }^{[31,32]}$. Perturbations in JNK activation result in failed osteoclastogenesis ${ }^{[33]}$. p38 is involved in controlling osteoclast differentiation but not osteoclast function [34]. In our study, we found that Luteoloside attenuated the phosphorylation of ERK and p38, but not the phosphorylation of JNK. In addition, MEK1 in combination with ERK signaling can influence NF- $\mathrm{B}$ signaling by enhancing IKK activity and also induce c-Fos and NFATc1 protein ${ }^{[35-37]}$. Similarly, p38 MAPK upstream components transforming growth factor-beta-activated kinase 1 (TAK1) and mitogen-activated protein kinase kinase 6 (MKK6) cooperate with NF- $\kappa B$ signaling by enhancing p65/Rel A nuclear translocation and NFATc1 in RANKL-induced osteoclastogenesis $[34,38]$. Collectively, Luteoloside inhibits multiple RANKL-induced signaling pathways, which leads to the inhibition of osteoclasto formation and function in vitro.

Particle-induced osteolysis in TJA can lead to aseptic loosening and is thought to be a primary reason of implant failure ${ }^{[2,3]}$. Bacterial endotoxin is at least partially responsible for the biological response mediated by implant-derived wear particles ${ }^{[5]}$. Identification of agents that can inhibit particle-mediated osteolysis will reduce the adverse side effects associated with particle-induced aseptic loosening. Luteoloside is a bioactive natural compound with potent application in diseases associated with excessive inflammation $^{[12,13]}$. In this study, LPS-mediated bone destruction was markedly impaired by Luteoloside treatment, accompanied by reduced number of 
TRAcP-positive multinucleated osteoclasts, suggesting that Luteoloside may serve as anti-resorptive agent in vivo.

Taken together, our studies indicate that Luteoloside inhibited RANKL-induced osteoclast formation and function by suppression of NFATc1 activity and $\mathrm{Ca}^{2+}$ signaling, as well as NF- $\mathrm{BB}$ and MAPK pathways. Furthermore, Luteoloside protected against LPS-induced osteolysis in vivo, congruent with its effects in vitro. Therefore, Luteoloside has potential therapeutic effects against osteoclast-related osteolytic diseases such as periprosthetic and inflammatory osteolysis.

\section{Disclosures}

The authors declare no conflicts of interest.

\section{Acknowledgements}

The authors acknowledge the support from Australian Health and Medical Research Council (NHMRC No: APP1107828, APP1127396, APP1127156), Arthritis Foundation of Australia (The H J \&G J Mckenzie grant), Western Australia Medical \& Health Research Infrastructure Fund, University of Western Australia Research Collaboration Awards and Innovation Project of Guangxi Graduate Education. This study is also sponsored in part by Co-innovation Centre for Bio-Medicine, Guangxi Key Laboratory of Regenerative Medicine, Guangxi Medical University, and by the Natural Science Foundation of Guangxi Province (2015GXNSDA139019). Fangming Song and Jiake Xu made mutual collaborative visits in 2016.

\section{Authors' contribution}

Authors' roles: Fangming Song performed cell culture, RANKL and signaling studies, as well as data analysis; Chengming Wei performed LPS-induced osteolysis, Micro-CT scanning, Histological and histomorphometric analysis; Lin Zhou performed NF- $\kappa$ B and NFAT luciferase reporter gene assays. Mingli Yang performed the cell proliferation assay (MTS) analysis and confocal images of osteoclasts. Yuanjiao Huang contributed to the supply of natural compounds. Fangming Song wrote the paper; Jiake Xu and Jinmin Zhao designed and supervised the overall study. Jiake Xu, Jennifer Tickner and Qin An revised the manuscript. 


\section{References}

1. Harris WH. Wear and periprosthetic osteolysis: the problem. Clin Orthop Relat Res. 2001(393):66-70.

2. Abu-Amer Y, Darwech I and Clohisy JC. Aseptic loosening of total joint replacements: mechanisms underlying osteolysis and potential therapies. Arthritis Research \& Therapy. 2007; 9(1):1-7.

3. Dattani R. Femoral osteolysis following total hip replacement. Postgraduate Medical Journal. 2007; 83(979):312-316.

4. Greenfields EM, Bi Y, Ragab AA, Goldberg VM and Van De Motter RR. The role of osteoclast differentiation in aseptic loosening. Journal of Orthopaedic Research. 2002; 20(1):1-8.

5. Bi Y, Seabold JM, Kaar SG, et al. Adherent Endotoxin on Orthopedic Wear Particles Stimulates Cytokine Production and Osteoclast Differentiation. Journal of Bone and Mineral Research. 2001; 16(11):2082-2091.

6. Greenfield EM, Bi Y, Ragab AA, et al. Does endotoxin contribute to aseptic loosening of orthopedic implants? Journal of Biomedical Materials Research Part B: Applied Biomaterials. 2005; 72B(1):179-185.

7. Yip KHM, Zheng MH, Feng HT, et al. Sesquiterpene Lactone Parthenolide Blocks Lipopolysaccharide-Induced Osteolysis Through the Suppression of NF-kB Activity. Journal of Bone and Mineral Research. 2004; 19(11):1905-1916.

8. Zhai ZJ, Li HW, Liu GW, et al. Andrographolide suppresses RANKL-induced osteoclastogenesis in vitro and prevents inflammatory bone loss in vivo. British Journal of Pharmacology. 2014; 171(3):663-675.

9. Poltorak A, He X, Smirnova I, et al. Defective LPS signaling in C3H/HeJ and C57BL/10ScCr mice: mutations in Tlr4 gene. Science. 1998; 282(5396):2085-8.

10. Qureshi ST, Lariviere L, Leveque G, et al. Endotoxin-tolerant mice have mutations in Toll-like receptor 4 (Tlr4). J Exp Med. 1999; 189(4):615-25.

11. Nakashima S, Oda Y, Nakamura S, et al. Inhibitors of melanogenesis in B16 melanoma 4A5 cells from flower buds of Lawsonia inermis (Henna). Bioorganic \& Medicinal Chemistry Letters. 2015; 25(13):2702-2706.

12. Xiong J, Li S, Wang W, et al. Screening and identification of the antibacterial bioactive compounds from Lonicera japonica Thunb. leaves. Food Chemistry. 2013; 138(1):327-333.

13. Akram M, Syed Ahmed S, Kim K-A, et al. Heme oxygenase 1-mediated novel anti-inflammatory activities of Salvia plebeia and its active components. Journal of Ethnopharmacology. 2015; 174:322-330.

14. Cao Z, Ding Y, Ke Z, et al. Luteoloside Acts as 3C Protease Inhibitor of Enterovirus 71 In Vitro. PLoS One. 2016; 11(2):e0148693.

15. Feng H, Cheng T, Steer JH, et al. Myocyte enhancer factor 2 and microphthalmia-associated transcription factor cooperate with NFATc1 to transactivate the V-ATPase d2 promoter during RANKL-induced osteoclastogenesis. J Biol Chem. 2009; 284(21):14667-76.

16. Xu J, Tan JW, Huang L, et al. Cloning, sequencing, and functional characterization of the rat homologue of receptor activator of NF-kappaB ligand. J Bone Miner Res. 2000; 15(11):2178-86. 
17. Wang C, Steer JH, Joyce DA, et al. 12-O-tetradecanoylphorbol-13-acetate (TPA) inhibits osteoclastogenesis by suppressing RANKL-induced NF-kappaB activation. J Bone Miner Res. 2003; 18(12):2159-68.

18. van der Kraan AG, Chai RC, Singh PP, et al. HSP90 inhibitors enhance differentiation and MITF (microphthalmia transcription factor) activity in osteoclast progenitors. Biochem J. 2013; 451(2):235-44.

19. Boyle WJ, Simonet WS and Lacey DL. Osteoclast differentiation and activation. Nature. 2003; 423(6937):337-342.

20. Kobayashi N, Kadono Y, Naito A, et al. Segregation of TRAF6-mediated signaling pathways clarifies its role in osteoclastogenesis. EMBO J. 2001; 20(6):1271-80.

21. Grigoriadis AE, Wang ZQ, Cecchini MG, et al. c-Fos: a key regulator of osteoclast-macrophage lineage determination and bone remodeling. Science. 1994; 266(5184):443-8.

22. Takayanagi H, Kim S, Koga T, et al. Induction and activation of the transcription factor NFATc1 (NFAT2) integrate RANKL signaling in terminal differentiation of osteoclasts. Dev Cell. 2002; 3(6):889-901.

23. Asagiri M, Sato K, Usami T, et al. Autoamplification of NFATc1 expression determines its essential role in bone homeostasis. J Exp Med. 2005; 202(9):1261-9.

24. Balkan W, Martinez AF, Fernandez I, et al. Identification of NFAT binding sites that mediate stimulation of cathepsin K promoter activity by RANK ligand. Gene. 2009; 446(2):90-8.

25. Feng $\mathrm{H}$, Cheng $\mathrm{T}$, Steer $\mathrm{JH}$, et al. Myocyte enhancer factor 2 and microphthalmia-associated transcription factor cooperate with NFATc1 to transactivate the V-ATPase d2 promoter during RANKL-induced osteoclastogenesis. J Biol Chem. 2009; 284:(21):14667-76.

26. Negishi-Koga T and Takayanagi H. Ca2+-NFATc1 signaling is an essential axis of osteoclast differentiation. Immunol Rev. 2009; 231(1):241-56.

27. Xu J, Wu HF, Ang ESM, et al. NF-kappaB modulators in osteolytic bone diseases. Cytokine and Growth Factor Reviews. 2009; 20(1):7-17.

28. Iotsova V, Caamano J, Loy J, et al. Osteopetrosis in mice lacking NF-kappaB1 and NF-kappaB2. Nat Med. 1997; 3(11):1285-9.

29. Vaira S, Alhawagri M, Anwisye I, et al. RelA/p65 promotes osteoclast differentiation by blocking a RANKL-induced apoptotic JNK pathway in mice. J Clin Invest. 2008; 118(6):2088-97.

30. Baud'huin M, Duplomb L, Ruiz Velasco C, et al. Key roles of the OPG-RANK-RANKL system in bone oncology. Expert Rev Anticancer Ther. 2007; 7(2):221-32.

31. Nakamura H, Hirata A, Tsuji T and Yamamoto T. Role of osteoclast extracellular signal-regulated kinase (ERK) in cell survival and maintenance of cell polarity. J Bone Miner Res. 2003; 18(7):1198-205.

32. Hotokezaka H, Sakai E, Kanaoka K, et al. U0126 and PD98059, specific inhibitors of MEK, accelerate differentiation of RAW264.7 cells into osteoclast-like cells. J Biol Chem. 2002; 277(49):47366-72.

33. Ikeda F, Nishimura R, Matsubara T, et al. Critical roles of c-Jun signaling in regulation of NFAT family and RANKL-regulated osteoclast differentiation. J Clin Invest. 2004; 114(4):475-84.

34. Li X, Udagawa $\mathrm{N}$, Itoh $\mathrm{K}$, et al. p38 MAPK-mediated signals are required for inducing 
osteoclast differentiation but not for osteoclast function. Endocrinology. 2002; 143(8):3105-13.

35. Miyazaki T, Katagiri H, Kanegae Y, et al. Reciprocal role of ERK and NF-kappaB pathways in survival and activation of osteoclasts. J Cell Biol. 2000; 148(2):333-42.

36. Monje P, Hernandez-Losa J, Lyons RJ, Castellone MD and Gutkind JS. Regulation of the transcriptional activity of c-Fos by ERK. A novel role for the prolyl isomerase PIN1. J Biol Chem. 2005; 280(42):35081-4.

37. Wada $\mathrm{T}$ and Penninger JM. Mitogen-activated protein kinases in apoptosis regulation. Oncogene. 2004; 23(16):2838-2849.

38. Zarubin $\mathrm{T}$ and Han J. Activation and signaling of the p38 MAP kinase pathway. Cell Res. 2005; 15(1):11-8. 


\section{Figure legends}

Fig. 1. Luteoloside inhibits RANKL-induced osteoclastogenesis in vitro.

(A) Chemical structure of Luteoloside. (B) Representative light microscope images of RANKL-induced osteoclast formation treated with indicated concentrations of Luteoloside for 5 days (Magnification = 100X; scale bar=100 $\mu \mathrm{m}) .(C)$ Quantitative analysis of TRAcP staining images relative to RANKL-induced controls. $(D)$ Low power image showing the effect of indicated concentrations of Luteoloside treatment. (E) Quantification of TRAcP-positive multinucleated cells (nuclei>3) treated with indicated concentrations of Luteoloside $(n=3)$. $(F)$ Low power image showing the effect of $10 \mu \mathrm{M}$ Luteoloside treatment on indicated days. $(G)$ Quantification of TRAcP-positive multinucleated cells (nuclei>3) treated with $10 \mu \mathrm{M}$ Luteoloside on indicated days $(\mathrm{n}=3)$. $(H)$ Representative confocal images of osteoclasts stained for F-actin and nuclei; untreated osteoclasts (a-c), osteoclasts treated with $5 \mu \mathrm{M}$ Luteoloside (d-f), osteoclasts treated with $10 \mu \mathrm{M}$ Luteoloside (g-i). (I) Effect of indicated concentrations Luteoloside on viability of BMMs as measured by MTS assay. (n=3). "-" means RANKL untreated; "+" means RANKL treated. ${ }^{*} p<0.05,{ }^{* *} p<0.01,{ }^{* * *} p<0.001$ relative to RANKL-induced controls.

Fig. 2. Luteoloside suppresses RANKL-induced osteoclast function and osteoclastic genes expression.

(A) Representative images of hydroxyapatite resorption (left) and TRAcP stained osteoclasts (right) on hydroxyapatite coated surfaces (Magnification $=4 \mathrm{X}$; scale bar=500 $\mu \mathrm{m}$ ). $(B)$ Quantification of the number of TRAcP positive multinucleated cells (nuclei $>3$ ). $(n=3)$. (C) Quantification of the percentage area of hydroxyapatite surface resorbed per osteoclast. $(\mathrm{n}=3)$. ( $(D)$ Real-time PCR analysis was performed to detect osteoclast-specific genes TRAcP, MMP9, Calcitonin receptor, CTSK, NFATc1 and V-ATPase d2. The expression levels of these genes were normalized to the expression of GAPDH. ${ }^{*} p<0.05,{ }^{* *} p<0.01,{ }^{* * *} p<0.001$ relative to RANKL-induced controls.

Fig. 3. Luteoloside abrogates RANKL induced calcium oscillation and associated NFATc1 activity.

(A) RAW 264.7 cells transfected with an NFAT luciferase construct were pre-treated with indicated concentrations of Luteoloside, followed by GST-rRANKL (50 ng/mL) stimulation for 24 hours. NFAT luciferase activity was measured using a luciferase reporter assay system. (n=3). (B) Representative images of Western blots demonstrating the effect of Luteoloside on c-Fos, NFATc1, and V-ATPase-d2 induced by RANKL. Cell extracts were prepared from BMMs pre-treated with Luteoloside $(10 \mu \mathrm{M})$ for 1 hour and subsequently activated by GST-rRANKL (50 ng/mL) for the indicated times. (C-E) The ratios of intensity of c-Fos $(C)$, NFATc1 $(D)$, and V-ATPase-d2 $(E)$ relative to $\beta$-actin were determined using Image J. $(\mathrm{n}=3)$. $(F-H)$ Representative images of $\mathrm{Ca}^{2+}$ oscillation pattern stimulated by RANKL $(F)$, negative control (M-CSF only) $(G), 10 \mu \mathrm{M}$ Luteoloside treatment prior to RANKL stimulation $(H)$. (I) Quantification of intensity of $\mathrm{Ca}^{2+}$ oscillation captured across multiple cells for each condition and maximum peak intensity minus baseline intensity. ( $\mathrm{n}>16$ individual cells/well, 3 wells/treatment). ( $\mathrm{n}=3$ ). " -" means RANKL untreated; "+" means RANKL treated. ${ }^{*} p<0.05$, $* * p<0.01$, $* * * p<0.001$ relative to RANKL-induced controls.

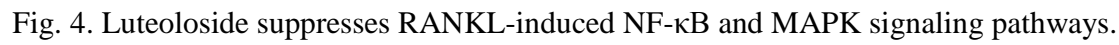

(A) Representative Western blot images of the effect of Luteoloside on degradation of IкB- $\alpha$ induced by RANKL. Cell extracts were prepared from BMMs pre-treated with Luteoloside $(10 \mu \mathrm{M})$ for 1 hour and subsequently

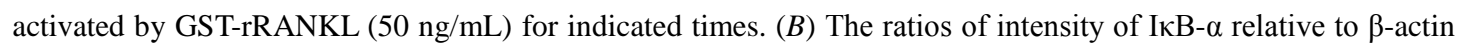
were determined using Image J. (n=3). (C) RAW 264.7 cells transfected with an NF-kB luciferase construct were pre-treated with indicated concentrations of Luteoloside for 1 hour, followed by GST-rRANKL stimulation (50 
$\mathrm{ng} / \mathrm{mL})$ for 8 hours. NF- $\mathrm{kB}$ Luciferase activity was measured using luciferase reporter assay system. $(\mathrm{n}=3)$. $(D)$ Representative images of RANKL-induced p65 nuclear translocation; i) no RANKL, ii) RANKL, iii) RANKL + $10 \mu \mathrm{M}$ Luteoloside, iv) negative control (absence of p65 antibody). Black arrow indicates p65 nuclear translocation, white arrow indicates cytoplasmic p65. (E) Quantification of the percentage of cells showing p65 nuclear translocation. $\mathrm{n}=3$. $(F)$ Representative Western blot images of the effect of Luteoloside on phosphorylation of ERK, JNK, p38 induced by RANKL. Cell extracts were prepared from BMMs pre-treated with Luteoloside (10 $\mu \mathrm{M})$ for 1 hour and subsequently stimulated with GST-rRANKL (50 ng/mL) for indicated times. (G-I) The ratios of intensity of p-ERK relative to total ERK $(G)$, p-JNK relative to total JNK $(H)$, and p-p38 relative to total p38 $(I)$ were determined using Image J. $\mathrm{n}=3$. " -" means RANKL untreated; "+" means RANKL treated. ${ }^{*} p<0.05$, ${ }^{* *} p<0.01,{ }^{* * *} p<0.001$ relative to RANKL-induced controls.

Fig. 5. Luteoloside protects against LPS-induced bone loss in vivo.

(A) Micro-CT scanning and 3D reconstruction of whole calvaria from Sham group (PBS; control), Vehicle group (LPS; $5 \mathrm{mg} / \mathrm{kg}$ body weight), LPS with low dose Luteoloside $(1 \mathrm{mg} / \mathrm{kg}$ ) and LPS with high dose Luteoloside (2 $\mathrm{mg} / \mathrm{kg}$ ). (B-D) Quantitative analysis of bone volume/total volume (BV/TV) (B), the number of pores $(C)$ and percentage porosity $(D) .(\mathrm{n}=5) ;{ }^{*} \mathrm{p}<0.05,{ }^{* *} \mathrm{p}<0.01,{ }^{* * *} \mathrm{p}<0.001$ relative to Vehicle.

Fig. 6. Histological and histomorphometric analysis of the effect of Luteoloside on LPS-induced bone loss in vivo. (A) Representative images of calvaria stained with H\&E and TRAcP from Sham group (PBS; control), Vehicle group (LPS; $5 \mathrm{mg} / \mathrm{kg}$ body weight), LPS with low dose Luteoloside ( $1 \mathrm{mg} / \mathrm{kg}$ ) and LPS with high dose Luteoloside (2 mg/kg). Black arrows indicate TRAcP positive cells. (B-D) Quantification of BV/TV (B), TRAcP (+) Cell Number $(C)$ and TRAcP $(+)$ Cell No/BS $(D) . \mathrm{n}=3 ;{ }^{*} \mathrm{p}<0.05,{ }^{* *} \mathrm{p}<0.01,{ }^{* * *} \mathrm{p}<0.001$ relative to Vehicle. 